\title{
The First Galaxy Cluster Discovered by the VISTA Variables in the Vía Láctea Survey
}

\author{
L. D. Baravalle ${ }^{1,2}$, J. L. Nilo Castellón ${ }^{3,4}$, M. V. Alonso ${ }^{1,2}$ (1) J. Díaz Tello ${ }^{5,6}$, G. Damke ${ }^{4,7}$, C. Valotto ${ }^{1,2}$, H. Cuevas Larenas ${ }^{3}$,

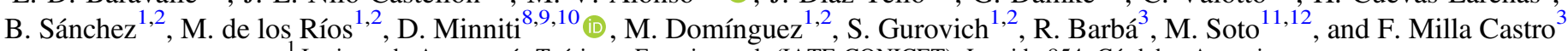 \\ ${ }_{1}$ Instituto de Astronomía Teórica y Experimental, (IATE-CONICET), Laprida 854, Córdoba, Argentina \\ ${ }^{2}$ Observatorio Astronómico de Córdoba, Universidad Nacional de Córdoba, Laprida 854, Córdoba, Argentina \\ ${ }^{3}$ Departamento de Física y Astronomía, Facultad de Ciencias, Universidad de La Serena, Av. Juan Cisternas 1200 Norte, La Serena, Chile \\ ${ }^{4}$ Instituto de Investigación Multidisciplinario en Ciencia y Tecnología, Universidad de La Serena. Avenida Juan Cisternas 1400, La Serena, Chile \\ ${ }^{5}$ Preuniversitario UC, Pontificia Universidad Católica de Chile, Chile \\ ${ }^{6}$ Instituto de Astronomía, Sede Ensenada, Universidad Nacional Autónoma de México, México \\ 7 AURA Observatory in Chile, Avenida Juan Cisternas 1500, La Serena, Chile \\ ${ }^{8}$ Millennium Institute of Astrophysics, Chile \\ ${ }^{9}$ Departamento de Ciencias Físicas, Universidad Andrés Bello, República 220, Santiago, Chile \\ ${ }^{10}$ Vatican Observatory, Vatican City State V-00120, Italy \\ ${ }^{11}$ Instituto de Astronomía y Ciencias Planetarias de Atacama, Universidad de Atacama, Copayapu 485, Copiapó, Chile \\ ${ }^{12}$ Space Telescope Science Institute, 3700 San Martin Drive, Baltimore, MD 21218, USA \\ Received 2018 July 27; revised 2018 November 5; accepted 2019 January 15; published 2019 March 20
}

\begin{abstract}
We report the first confirmed detection of the galaxy cluster VVV-J144321-611754 at very low latitudes ( $\left.l=315^{\circ} .836, b=-1^{\circ} .650\right)$ located in the tile d015 of the VISTA Variables in the Vía Láctea (VVV) survey. We defined the region of $30 \times 30 \operatorname{arcmin}^{2}$ centered in the brightest galaxy finding 25 galaxies. For these objects, extinction-corrected median colors of $\left(H-K_{s}\right)=0.34 \pm 0.05 \mathrm{mag},(J-H)=0.57 \pm 0.08 \mathrm{mag}$, and $\left(J-K_{s}\right)=$ $0.87 \pm 0.06 \mathrm{mag} ; R_{1 / 2}=1.59 \pm 0$ " $16 ; C=3.01 \pm 0.08$; and Sérsic index $n=4.63 \pm 0.39$ were estimated. They were visually confirmed showing characteristics of early-type galaxies in the near-IR images. An automatic clustering analysis performed in the whole tile found that the concentration of galaxies VVV-J144321-611754 is a real, compact concentration of early-type galaxies. Assuming a typical galaxy cluster with low X-ray luminosity, the photometric redshift of the brightest galaxy is $z=0.196 \pm 0.025$. Follow-up near-IR spectroscopy with FLAMINGOS-2 at the Gemini-South telescope revealed that the two brighter cluster galaxies have typical spectra of early-type galaxies and the estimated redshift for the brightest galaxy VVV-J144321.06-611753.9 is $z=0.234 \pm 0.022$ and that for VVV-J144319.02-611746.1 is $z=0.232 \pm 0.019$. Finally, these galaxies clearly follow the cluster red sequence in the rest-frame near-IR color-magnitude diagram with a slope similar to a galaxy cluster at a redshift of 0.2 . These results are consistent with the presence of a bona fide galaxy cluster beyond the Milky Way disk.
\end{abstract}

Key words: galaxies: clusters: general - surveys - infrared: galaxies

\section{Introduction}

Extragalactic sources and large-scale structure behind the Milky Way (MW) are obscured by dust and stellar crowding. The light of these sources is dimmed more than $\sim 25 \%$ and $\sim 10 \%$ in the optical and near-IR wavelengths, respectively (Henning et al. 1998). This effect is far larger close to the MW midplane because the dust is concentrated there.

Several efforts have been made to study the distribution of galaxies behind the MW. Kraan-Korteweg \& Lahav (2000) have reviewed the search of galaxies in the Zone of Avoidance (ZoA, $|b|<20^{\circ}$ ) using optical, near-IR, far-IR, radio, and X-ray wavelengths. In the near-IR, the Two Micron All Sky Survey (2MASS) Redshift Survey (Huchra et al. 2012), 97.6\% complete to the limiting magnitude of $K_{s}=11.75 \mathrm{mag}$, was used to generate the catalog of groups of galaxies (Tully 2015) in the ZoA. At radio wavelengths, we can mention the Arecibo $L$-band Feed Array ZoA survey (McIntyre et al. 2015) and the blind $21 \mathrm{~cm} \mathrm{H}$ I-line imaging survey on the Perseus-Pisces Supercluster filament crossing the ZoA (Ramatsoku et al. 2016). The results are complementary: near-IR selection favors the detection of early-type galaxies, and blind $\mathrm{H}$ I surveys favor the detection of late-type galaxies typically.

However, rich galaxy clusters are traced by X-ray emissions of the diffuse gas present in the systems. A search for clusters of galaxies in the ZoA was carried out by Ebeling et al. (2002) using the ROSAT All Sky Survey Bright Source Catalog (Ebeling et al. 1996; Voges et al. 1999). There are only 13 galaxy clusters with measured redshifts in the literature at lower galactic latitudes $\left(|b|<2^{\circ}\right)$ : 1 cluster detected from $X M M$ Newton observations (Nevalainen et al. 2001), 2 clusters from the Clusters in the Zone of Avoidance project (Ebeling et al. 2002), 1 from the meta-catalog of X-ray-detected clusters of galaxies (Piffaretti et al. 2011), and 9 from the Planck catalog of Sunyaev-Zeldovich sources (Ma et al. 2013).

The VISTA Variables in the Vía Láctea (Minniti et al. 2010, hereafter VVV) is a public ESO photometric variability survey aimed mainly at studying the stellar populations of the MW bulge $\left(-10^{\circ}<\ell<+10^{\circ}\right.$ and $\left.-10^{\circ}<b<+5^{\circ}\right)$ and MW disk $\left(-65^{\circ}<\ell<-10^{\circ}\right.$ and $\left.-2^{\circ}<b<+2^{\circ}\right)$ in $Z, Y, J, H$, and $K_{s}$ near-IR passbands. This survey has yielded the detection of new globular clusters (Minniti et al. 2011, 2017), new stellar open clusters (Borissova et al. 2011, 2014; Barbá et al. 2015), the discovery of brown dwarfs (Beamín et al. 2013), and other classes of variable stars. Moreover, data from the VVV survey have also been used for extragalactic studies. Amôres et al. (2012) reported 204 candidate galaxies behind the Galactic disk, in the direction of Carina's spiral arm $\left(l \sim 285^{\circ}\right.$ to $290^{\circ}$ ) based on visual inspection. Coldwell et al. (2014) used near-IR photometry to confirm the existence of the previously 

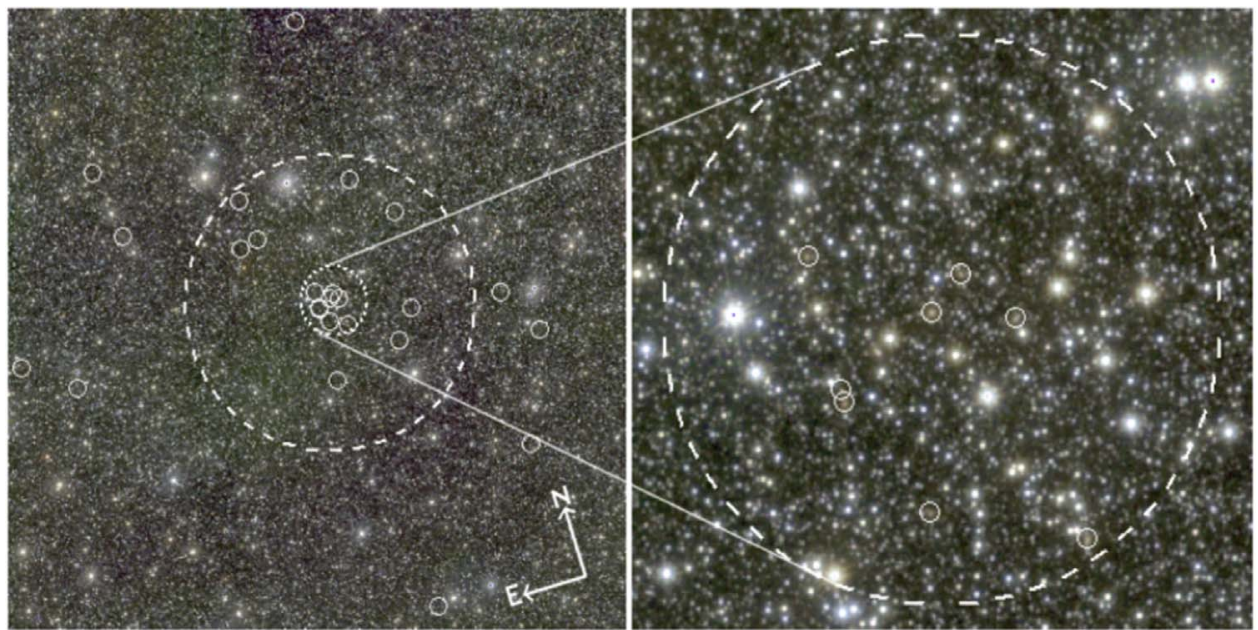

Figure 1. Color images of the concentration of galaxies showing the region of $30 \times 30 \operatorname{arcmin}^{2}$ (left panel) and the central parts of $3 \times 3 \operatorname{arcmin}^{2}($ right panel). The 25 visually confirmed galaxies are surrounded with small circles. The circles with diameters of 3 and 7 arcmin are shown with dashed lines.

X-ray-detected galaxy cluster Suzaku J1759-3450 (Mori et al. 2013). Coldwell et al. (2014) found 15 galaxies within a projected distance of $350 \mathrm{kpc}$ from the central X-ray peak emission at a redshift of 0.13 . More recently, Baravalle et al. (2018) presented a method to search and characterize extragalactic sources detected from VVV image data. They derived photometric and morphological parameters of the sources by combining SExtractor+PSFEx.

In this study, we use our photometric procedure to search for extragalactic sources and present the analysis for a galaxy cluster candidate found in the VVV tile d015. VVV$\mathrm{J} 144321.06-611753.9$ is the first confirmed galaxy cluster selected originally through VVV photometry. This paper is organized as follows: In Section 2, we describe the near-IR data and selection criteria, both for the VVV photometry and for the FLAMINGOS-2 spectroscopic data obtained at Gemini-South. In Section 3, we present the cluster detection and analysis from four different methods, all used to confirm that VVVJ144321.06-611753.9 is a bona fide galaxy cluster. The methods are (1) automatic clustering analysis, (2) spectral energy distribution (SED) modeling, (3) spectroscopic redshift measurements, and (4) the cluster red sequence. In Section 4, we present a summary of the data analysis and main results. Throughout the paper, we use the following cosmological parameters: $H_{0}=70.4 \mathrm{~km} \mathrm{~s}^{-1} \mathrm{Mpc}^{-1}, \quad \Omega_{M}=0.272, \quad$ and $\Omega_{\lambda}=0.728$ (Komatsu et al. 2011).

\section{The Data}

A concentration of extended objects was detected serendipitously in the search for new stellar cluster candidates in the VVV survey (Barbá et al. 2015). VVV-J144321.06-611753.9 was found in the d015 MW disk tile centered at J2000 R.A. $=14^{\mathrm{h}} 43^{\mathrm{m}} 42^{\mathrm{s}} \cdot 14$, decl. $=-61^{\circ} 40^{\prime} 33^{\prime \prime} \cdot 96 \quad\left(l=315^{\circ} .836\right.$, $b=-1^{\circ} .650$ ), which corresponds to the brightest object coordinates.

\subsection{VVV Near-IR Photometry}

In order to detect and characterize the extragalactic sources in the tile d015, we applied the photometric methodology developed in Baravalle et al. (2018), which uses the combination of SExtractor v2.19.1 and PSFEx v3.17. The sources were selected using the stellar index CLASS_STAR, the
SPREAD_MODEL $(\Phi)$ parameter, the radius that contains $50 \%$ of the total flux of an object $\left(R_{1 / 2}\right)$, the concentration index (C), and the near-IR colors. The adopted criteria consider the following: CLASS_STAR $<0.3, \quad 1$ !" $0<R_{1 / 2}<5$ !" 0 , $2.1<\mathrm{C}<5$, and $\Phi>0.002$, and the colors $0.5 \mathrm{mag}<$ $\left(J-K_{s}\right)<2.0 \mathrm{mag}, 0.0 \mathrm{mag}<(J-H)<1.0 \mathrm{mag}$, and 0.0 mag $<\left(H-K_{s}\right)<2.0 \mathrm{mag}$, with the color restriction $(J-H)+$ $0.9\left(H-K_{s}\right)>0.44 \mathrm{mag}$ to minimize false detections. Also, the sources with $Z$ and $Y$ detections should satisfy $-0.3 \mathrm{mag}<$ $(Y-J)<1.0 \mathrm{mag}$ and $-0.3 \mathrm{mag}<(Z-Y)<1.0 \mathrm{mag}$.

Following these criteria, we detected 933 extragalactic sources in the d015 VVV MW disk tile, with 24 located in the region of $30 \times 30 \operatorname{arcmin}^{2}$ centered at VVV-J144321.05611753.9. Three objects were discarded because they are low surface brightness sources with strong stellar contamination that could not be effectively corrected. The objects VVVJ144243.0-611529.8, VVV-J144331.6-612133.1, and VVVJ144426.4-611101.3 have contamination by nearby stars, and they were corrected using the procedure described in Baravalle et al. (2018). Additionally, four sources that satisfied the above criteria but had slightly smaller $R_{1 / 2}$ than 1 !" were also included after a visual inspection. Our final sample consists of 25 extragalactic sources, all of them visually confirmed to be galaxies. They were all detected in the $J, H$, and $K_{s}$ passbands, and only four sources were also detected in the $Z$ and $Y \mathrm{VVV}$ passbands. Figure 1 shows a cutout made from a composite $(J$, $H$, and $K_{s}$ ) color image of the field showing the concentration of galaxies, centered at the brightest galaxy (VVV-J144321.05611753.9). The 25 galaxies of the concentration are marked by small circles. The left panel corresponds to a $30 \times 30 \mathrm{arcmin}^{2}$ box identifying with dashed lines two circles with diameters of 3 and 7 arcmin. The right panel shows the $3 \times 3 \operatorname{arcmin}^{2}$ central parts of the cluster candidate.

Table 1 shows the photometric properties of the 25 galaxies. Column (1) gives the VVV identification; Columns (2) and (3) give RA and decl. in J2000 coordinates; Columns (4) to (8) show the point-spread function (PSF) $Z, Y, J, H$, and $K_{s}$ magnitudes; Columns (9) to (13) show the aperture magnitudes within a 2 arcsec diameter, respectively; and finally, Columns (14) to (17) list the morphological parameters based on $K_{s}$-band photometry: the half-light radius, concentration index, ellipticity, and Sérsic index. The PSF magnitudes are estimates of the total $K_{s}$ magnitudes, and they range between 13.70 and 
Table 1

Photometric and Morphological Parameters of the Galaxies in the Galaxy Concentration

\begin{tabular}{|c|c|c|c|c|c|c|c|c|c|c|c|c|c|c|c|c|}
\hline ID & R.A. (J2000) & Decl. (J2000) & $\overline{Z Z}$ & $Y$ & 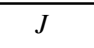 & $H$ & $\overline{K_{s}}$ & $\overline{Z Z_{2^{\prime \prime}}}$ & $\overline{Y_{2^{\prime \prime}}}$ & $\overline{J_{2^{\prime \prime}}}$ & $\overline{H_{2^{\prime \prime}}}$ & 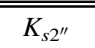 & $R_{1 / 2}$ & $\bar{C}$ & $\overline{\epsilon \epsilon}$ & $n$ \\
\hline VVV-J144217.23-612102.9 & $14: 42: 17.23$ & $-61: 21: 02.9$ & 15.85 & 15.58 & 15.45 & 15.16 & 14.95 & 15.79 & 15.47 & 15.27 & 14.96 & 14.74 & 1.27 & 2.79 & 0.29 & 2.09 \\
\hline VVV-J144243.03-611529.8 & $14: 42: 43.03$ & $-61: 15: 29.8$ & $\ldots$ & $\ldots$ & 14.87 & 14.16 & 13.79 & $\ldots$ & $\ldots$ & 14.57 & 13.83 & 13.53 & 2.16 & 3.80 & 0.16 & 3.45 \\
\hline VVV-J144252.33-611955.9 & $14: 42: 52.33$ & $-61: 19: 55.9$ & $\ldots$ & $\ldots$ & 16.52 & 15.96 & 15.61 & $\ldots$ & $\ldots$ & 15.76 & 16.32 & 15.46 & 0.78 & 2.55 & 0.25 & 4.81 \\
\hline VVV-J144254.41-611308.4 & $14: 42: 54.41$ & $-61: 13: 08.4$ & $\ldots$ & $\ldots$ & 16.63 & 15.96 & 15.53 & $\ldots$ & $\ldots$ & 16.5 & 15.84 & 15.39 & 1.10 & 2.8 & 0.34 & 4.63 \\
\hline VVV-J144302.30-612104.7 & $14: 43: 02.30$ & $-61: 21: 04.7$ & $\ldots$ & $\ldots$ & 15.58 & 15.12 & 14.80 & $\ldots$ & $\ldots$ & 15.37 & 14.95 & 14.61 & 1.01 & 3.01 & 0.07 & 3.36 \\
\hline VVV-J144317.50-611807.6 & $14: 43: 17.50$ & $-61: 18: 07.6$ & 14.64 & 14.71 & 14.62 & 14.30 & 14.06 & 14.65 & 14.62 & 14.40 & 14.07 & 13.85 & 1.79 & 3.39 & 0.34 & 3.39 \\
\hline VVV-J144318.67-611925.5 & $14: 43: 18.67$ & $-61: 19: 25.5$ & $\ldots$ & $\ldots$ & 15.03 & 14.43 & 13.89 & $\ldots$ & $\ldots$ & 14.63 & 13.91 & 13.88 & 2.65 & 2.97 & 0.46 & 2.67 \\
\hline VVV-J144319.03-611746.2 & $14: 43: 19.03$ & $-61: 17: 46.2$ & $\ldots$ & $\ldots$ & 15.09 & 14.55 & 14.10 & $\ldots$ & $\ldots$ & 15.33 & 14.55 & 14.26 & 1.08 & 2.92 & 0.21 & 6.07 \\
\hline VVV-J144321.05-611753.9 & $14: 43: 21.05$ & $-61: 17: 53.9$ & $\ldots$ & $\ldots$ & 14.60 & 14.07 & 13.73 & $\ldots$ & $\ldots$ & 14.56 & 13.95 & 13.60 & 2.33 & 3.49 & 0.15 & 7.15 \\
\hline VVV-J144325.01-611854.7 & $14: 43: 25.01$ & $-61: 18: 54.7$ & $\ldots$ & $\ldots$ & 15.76 & 15.09 & 14.81 & $\ldots$ & $\ldots$ & 15.59 & 14.93 & 14.64 & 1.95 & 3.48 & 0.17 & 5.18 \\
\hline VVV-J144325.32-611719.0 & $14: 43: 25.32$ & $-61: 17: 19.0$ & $\ldots$ & $\ldots$ & 15.16 & 14.57 & 14.22 & $\ldots$ & $\ldots$ & 15.02 & 14.48 & 14.09 & 1.73 & 3.25 & 0.30 & 4.63 \\
\hline VVV-J144326.47-611804.6 & $14: 43: 26.47$ & $-61: 18: 04.6$ & $\ldots$ & $\ldots$ & 15.40 & 14.67 & 14.47 & $\ldots$ & $\ldots$ & 15.3 & 14.61 & 14.43 & 0.95 & 3.81 & 0.22 & 9.11 \\
\hline VVV-J144326.58-611808.8 & $14: 43: 26.58$ & $-61: 18: 08.8$ & $\ldots$ & $\ldots$ & 15.67 & 14.95 & 14.53 & $\ldots$ & $\ldots$ & 15.35 & 14.65 & 14.19 & 1.59 & 2.89 & 0.45 & 2.39 \\
\hline VVV-J144331.64-612133.1 & $14: 43: 31.64$ & $-61: 21: 33.1$ & $\ldots$ & $\ldots$ & 15.81 & 15.24 & 14.94 & $\ldots$ & $\ldots$ & 15.10 & 15.60 & 14.81 & 0.82 & 2.71 & 0.09 & 6.89 \\
\hline VVV-J144337.61-611355.6 & $14: 43: 37.61$ & $-61: 13: 55.6$ & $\ldots$ & $\ldots$ & 16.31 & 15.72 & 15.37 & $\ldots$ & $\ldots$ & 15.61 & 16.14 & 15.21 & 0.86 & 2.74 & 0.53 & 5.83 \\
\hline VVV-J144338.11-611153.2 & $14: 43: 38.11$ & $-61: 11: 53.2$ & $\ldots$ & $\ldots$ & 14.68 & 14.13 & 13.83 & $\ldots$ & $\ldots$ & 14.50 & 13.94 & 13.65 & 1.43 & 3.01 & 0.41 & 2.64 \\
\hline VVV-J144345.26-611356.6 & $14: 43: 45.26$ & $-61: 13: 56.6$ & $\ldots$ & $\ldots$ & 15.87 & 15.57 & 15.54 & $\ldots$ & $\ldots$ & 15.81 & 15.39 & 15.23 & 1.92 & 3.10 & 0.44 & 2.48 \\
\hline VVV-J144208.98-612325.1 & $14: 42: 08.98$ & $-61: 23: 25.1$ & $\ldots$ & $\ldots$ & 16.55 & 15.86 & 15.37 & $\ldots$ & $\ldots$ & 16.31 & 15.67 & 15.22 & 1.04 & 2.87 & 0.48 & 6.62 \\
\hline VVV-J144426.62-610744.4 & $14: 44: 26.62$ & $-61: 07: 44.4$ & $\ldots$ & $\ldots$ & 15.11 & 14.51 & 14.29 & $\ldots$ & $\ldots$ & 15.12 & 14.4 & 14.19 & 1.42 & 3.46 & 0.08 & 5.90 \\
\hline VVV-J144230.74-612811.3 & $14: 42: 30.74$ & $-61: 28: 11.3$ & 15.21 & 15.86 & 15.39 & 15.11 & 14.77 & 15.91 & 15.86 & 15.59 & 15.34 & 14.97 & 2.3 & 2.39 & 0.29 & 2.66 \\
\hline VVV-J144331.39-613319.8 & $14: 43: 31.39$ & $-61: 33: 19.8$ & $\ldots$ & $\ldots$ & 16.99 & 16.28 & 16.0 & $\ldots$ & $\cdots$ & 16.75 & 16.18 & 15.88 & 1.33 & 2.83 & 0.39 & 4.09 \\
\hline VVV-J144248.72-610518.6 & $14: 42: 48.72$ & $-61: 05: 18.6$ & $\ldots$ & $\ldots$ & 15.81 & 15.12 & 14.64 & $\ldots$ & $\ldots$ & 15.91 & 14.92 & 14.515 & 2.71 & 3.29 & 0.65 & 4.31 \\
\hline VVV-J144426.38-611101.3 & $14: 44: 26.38$ & $-61: 11: 01.3$ & $\ldots$ & $\ldots$ & 15.5 & 13.9 & 13.9 & $\ldots$ & $\ldots$ & 14.07 & 13.49 & 13.7 & 2.44 & 3.48 & 0.21 & 1.74 \\
\hline VVV-J144507.49-611636.8 & $14: 45: 07.49$ & $-61: 16: 36.8$ & $\ldots$ & $\ldots$ & 16.05 & 15.71 & 15.41 & $\ldots$ & $\ldots$ & 16.11 & 15.58 & 15.19 & 4.09 & 3.43 & 0.68 & 4.67 \\
\hline VVV-J144524.77-611437.3 & $14: 45: 24.77$ & $-61: 14: 37.3$ & 15.86 & 15.34 & 14.7 & 14.06 & 13.72 & 15.86 & 15.26 & 14.6 & 14.17 & 13.641 & 3.06 & 3.76 & 0.44 & 7.37 \\
\hline
\end{tabular}


16.00 mag. All magnitudes were corrected for Galactic extinction using the maps of Schlafly \& Finkbeiner (2011) and the relative extinctions of Catelan et al. (2011). The completeness was estimated for the tiles d010 and d115 in Baravalle et al. (2018, Figure 3), showing that $80 \%$ completeness is reached for sources fainter than 17 to $17.5 K_{s}$ magnitudes. We are reaching these magnitude limits in the tile d015, with a median $K_{s}$ extinction value $A_{K s}=0.73 \pm$ $0.01 \mathrm{mag}$. In the studied region of $30 \times 30 \mathrm{arcmin}^{2}$, the extinction was not constant with median $A_{K s}=1.07 \pm$ 0.04 mag: $1.13 \pm 0.05 \mathrm{mag}$ in the central parts (radius smaller than 1.5), $1.12 \pm 0.07 \mathrm{mag}$ for a radius between 1.5 and 3!5, and $0.93 \pm 0.09 \mathrm{mag}$ in the most external parts.

The galaxies in the concentration are clearly small and red when compared against those in the whole tile. Extinctioncorrected median values of the photometric properties are PSF magnitudes, $J=15.50 \pm 0.13 \mathrm{mag}, H=15.09 \pm 0.14 \mathrm{mag}$, and $K_{s}=14.64 \pm 0.14 \mathrm{mag}$, and colors, $\left(H-K_{s}\right)=0.34 \pm$ $0.05 \mathrm{mag},(J-H)=0.57 \pm 0.08 \mathrm{mag}$, and $\left(J-K_{s}\right)=0.87 \pm$ 0.06 mag. The median morphological parameters are $R_{1 / 2}=$ $1.59 \pm 0$ ! $16, C=3.01 \pm 0.08, \epsilon=0.30 \pm 0.03$, and Sérsic index $n=4.63 \pm 0.39$.

\subsection{FLAMINGOS-2 IR Spectroscopy}

We obtained follow-up spectroscopic data using the nearinfrared imaging spectrograph FLAMINGOS-2 at GeminiSouth, in the Fast Turnaround (Program ID: GS-2016A-FT-18) observing mode. The observations were made during the nights of 2016 July 30 and August 2. We collected long-slit spectra with the $J H / H K$ grism and 0.72 slit. This resulted in a peak resolution $\mathrm{R} \sim 600$, a dispersion of $6.55 \AA \mathrm{pixel}^{-1}$, and a total spectral coverage from 0.98 to $1.80 \mu \mathrm{m}$. The projected on-sky slit length was 4.48 arcmin. We observed the eight brightest galaxies in the central part of the concentration. We set each pointing to include two objects per slit, constraining the angular separation between each galaxy pair to be lower than 3 arcmin. In addition, we optimized the slit position angle configuration or slit rotation to avoid bright stars for each setup. The objects have $K_{s}$ magnitudes between 13.7 and $14.8 \mathrm{mag}$, and the exposure time was $8 \times 120 \mathrm{~s}$ to reach a signal-to-noise ratio $(\mathrm{S} / \mathrm{N})>3$ for each combined galaxy spectrum. Calibration frames were acquired together with telluric standard-star spectra to remove telluric absorption features from the science data.

The spectra were reduced using the IRAF/Gemini tasks and the Gemini FLAMINGOS-2 PyRAF data reduction package. We performed the basic data reduction and combination of the 2D data with nsreduce and nscombine routines, respectively. The arc-line identification and wavelength calibration steps were carried out with the nsfitcoords and nstransform routines, respectively. After obtaining the 2D spectra, the 1D spectra were extracted via the nsextract routine, to then be telluriccorrected following the Maiolino et al. (1996) procedure. Finally, the spectra were also flux-calibrated using the photometric data available for the telluric standard star observed.

\section{Cluster Detection and Analysis}

In this section we present the photometric and spectroscopic results of the galaxy concentration VVV-J144321-611754. We performed an automatic clustering analysis in the tile d015 and obtained a photometric redshift estimate through the analysis of the SED. We determined the spectroscopic redshifts for the brightest objects and, finally, the cluster red sequence.

\subsection{Automatic Clustering Analysis}

We performed an automatic clustering analysis in order to gain insight about the nature of the galaxy clustering. This analysis aims to split the data into sets of associated objects with a joint conditional probability distribution. In our case, a mixture of simple Gaussian densities would separate the data into different groups by varying the number of free parameters, such as the number and shape of the Gaussians. The best model was obtained using the Bayesian information criterion, which penalizes those models with a high number of free parameters.

We used the public code Mclust (Scrucca et al. 2016) in the catalog of extragalactic sources found in the tile d015, applying the mixture of Gaussians to the sky coordinates, R.A. and decl. In order to use galaxies with higher probability of belonging to a galaxy cluster, they should satisfy $R_{1 / 2}<3$ arcsec, $n>2$, and $K_{s}$ magnitudes fainter than $15.7 \mathrm{mag}$. We found that the best model has four spherically symmetric Gaussians associated with four groups with different circular angular areas. Group 1 identified with the concentration of galaxies VVVJ144321-611754 has the smallest angular radius of the sample, 0.138 , whereas the other three groups have angular radii greater than 0.230 . Galaxies within this radius have a higher probability of belonging to the group. Figure 2 shows the distribution of the extragalactic sources of the tile d015 in galactic coordinates. The 25 galaxies of VVV-J144321-611754 are represented by small dots; the objects identified with groups 1 to 4 are respresented by open squares, circles, crosses, and triangles, respectively. The group angular sizes are shown in Figure 2. We also took into account some photometric properties of the extragalactic sources, such as the extinctioncorrected colors, $C$, and $n$ to characterize the groups. The median values for group 1 , which contains the concentration of galaxies, are $\left(J-K_{s}\right)=0.84 \pm 0.05 \mathrm{mag},\left(H-K_{s}\right)=0.30 \pm$ 0.04 mag, $\quad R_{1 / 2}=1.27 \pm 0 . " 09, \quad C=3.01 \pm 0.06, \quad \epsilon=$ $0.301 \pm 0.03$, and $n=4.3 \pm 0.3$. The other three groups include bluer galaxies with $C$ and $n$ higher than 3.03 and 4.75, respectively. Group 1 is formed with 24 extragalactic objects that were visually inspected; some of them are fainter galaxies with nearby bright stars or located at the outer parts with lower group probability. This result suggests that VVV-J144321611754 is a real concentration of galaxies formed by early-type objects.

\subsection{Modeling the SED}

EzGal (Mancone \& Gonzalez 2012) is a tool to model the evolution of the SED of a stellar population that gives some derived properties such as the magnitude evolution as a function of redshift for different passbands. This tool uses different stellar population synthesis (SPS) models, including Bruzual \& Charlot (2003), the Maraston model (Maraston 2005), the BaSTI model (Percival et al. 2009), and the Flexible Stellar Population Synthesis (FSPS; Conroy et al. 2009). The Bruzual \& Charlot (2003) model is the most commonly used, which computes the spectral evolution of stellar populations for different ages and a wide range of metallicities. The Maraston 


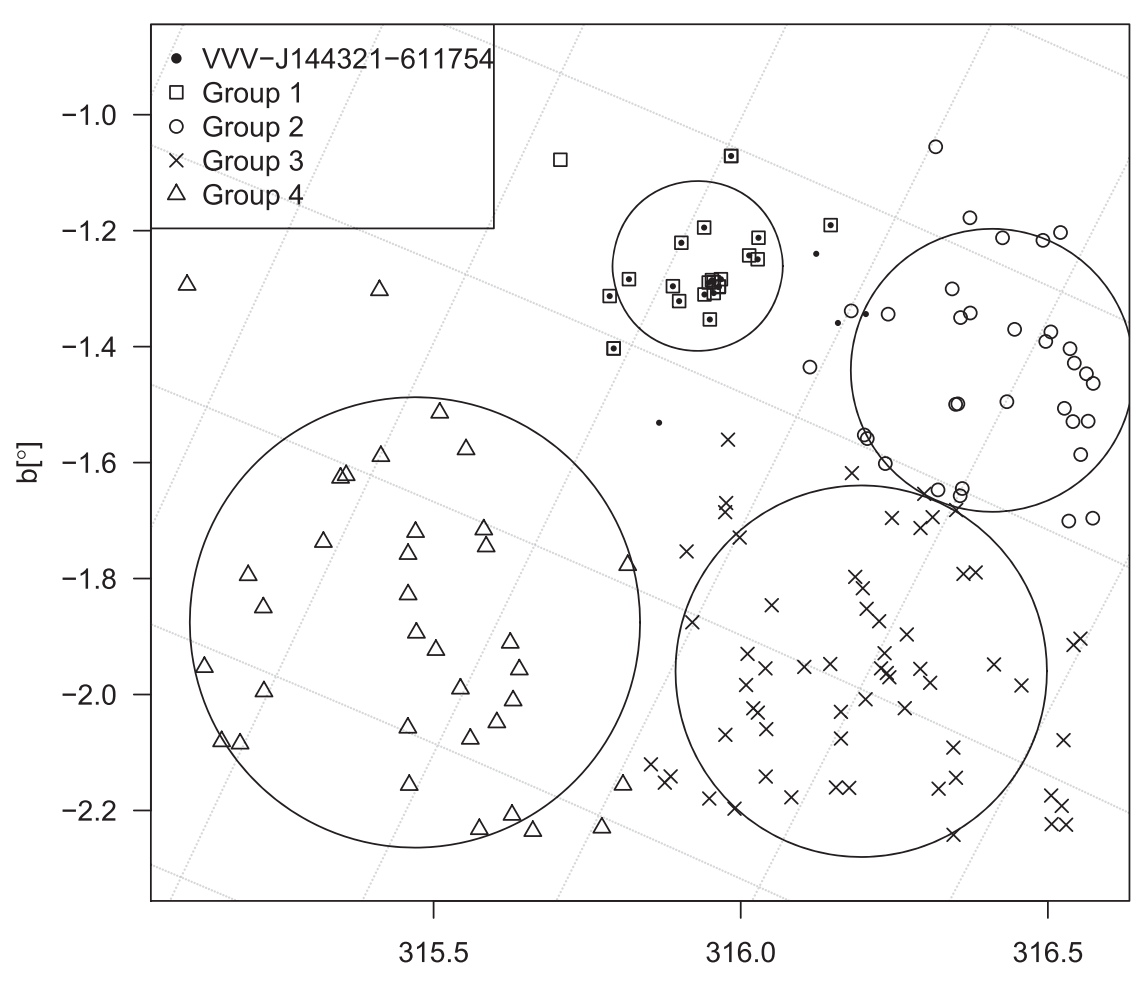

$\left[\left[^{\circ}\right]\right.$

Figure 2. Distribution of the extragalactic sources detected in the tile d015 in galactic coordinates. The groups obtained in the clustering analysis are represented by different symbols and encircled by the estimated angular size.

model includes a detailed treatment of thermally pulsating asymptotic giant branch stars that dominate the infrared light of a young stellar population. The BaSTI models include a wide range of metallicities for both solar-scaled and $\alpha$-enhanced metallicities. The FSPS models are unique in dealing with important SPS inputs, such as the initial mass function (IMF) or stellar evolution phases, treating them as free parameters, allowing the uncertainties introduced by various SPS inputs to be quantitatively measured.

The $K_{s}$ model magnitudes were obtained using EzGal for Bruzual \& Charlot (2003) models assuming a formation redshift of $z=3$, the Chabrier IMF, a solar metallicity, and a simple stellar population at different redshifts. We used a standard normalization of $K_{s}$ absolute magnitude of $-25.51 \pm 0.09$ (Brough et al. 2002) for redshifts higher than 0.1 assuming the cluster X-ray luminosity $L_{\mathrm{X}}(0.3-3.5 \mathrm{keV})<1.9 \times 10^{44} \mathrm{erg} \mathrm{\textrm {s } ^ { - 1 }}$. In the evolution of $K_{s}$ magnitudes, the brightest galaxy in the concentration has an observed $K_{s}$ magnitude of $13.77 \mathrm{mag}$, which corresponds to a photometric redshift of $0.196 \pm 0.025$. This value is also in agreement with the Stott et al. (2009) estimate based on the $K_{s}$ magnitude and color of the brightest cluster galaxy. Therefore, all these results indicate that the photometric redshift of the galaxy concentration VVV-J144321-611754 is $0.196 \pm 0.025$.

\subsection{Spectroscopic Redshifts}

The brighter galaxies VVV-J144321.06-611753.9 and VVVJ144319.02-611746.1 were observed in a single pointing. The spectra were flux-calibrated and then normalized to the photometry aperture fluxes, in order to be able to perform the dust reddening correction. The final spectra had $\mathrm{S} / \mathrm{N} \sim 9.5$, enough to obtain a precise redshift estimation. Figure 3 shows the spectra of the two galaxies. The solid lines represent the observed spectra with the continuum highly affected by the MW disk dust extinction, making the identification of the spectral features difficult. The dotted lines represent the spectra corrected for absorption of Galactic dust. The spectra were corrected by a simple model of dust reddening extinction in order to retrieve a hypothetical spectrum free of dust. We identified several molecular bands and absorption lines, such as the $\mathrm{TiO} \lambda 0.85 \mu \mathrm{m}$ and $\mathrm{ZrO} \lambda 0.93 \mu \mathrm{m}$ molecular bands overlapped with the $\mathrm{Ca}$ II and $\mathrm{Si}$ absorption lines, respectively. Similarly, the $\mathrm{CN}$ and $\mathrm{TiO}$ bands at $\lambda 1.1 \mu \mathrm{m}$ were identified, in addition to the blend of $\mathrm{C}_{2}, \mathrm{VO}$, and $\mathrm{FeH}$ bands at $\lambda 1.2 \mu \mathrm{m}$. Finally, we recognized the slump of $\mathrm{CN}$ and $\mathrm{H}_{2} \mathrm{O} \lambda 1.4 \mu \mathrm{m}$ bands. All these features, marked by vertical lines in the figure, are typical of old stellar populations (Rayner et al. 2009; Mason et al. 2015). The telluric absorption is shown as a shaded region. The estimated redshift for VVV-J144321.06-611753.9 is $z=0.234 \pm 0.022$, and that for VVV-J144319.02-611746.1 is $z=0.232 \pm 0.019$. The relative large uncertainties in these estimates are mainly related to the use of molecular bands. It was impossible to obtain redshifts for the fainter galaxies in the other pointings owing to the lower $\mathrm{S} / \mathrm{N}$ of the spectra.

For the two brightest galaxies, the spectra include features typical of early-type galaxies. Also, the good agreement between the two redshifts indicates that both galaxies likely belong to the same galaxy cluster. Also, for the brightest galaxy, the difference between the photometric and spectroscopic redshift estimates is about 0.044, and smaller than the uncertainties. We use this spectroscopic redshift as a cluster redshift estimate. 

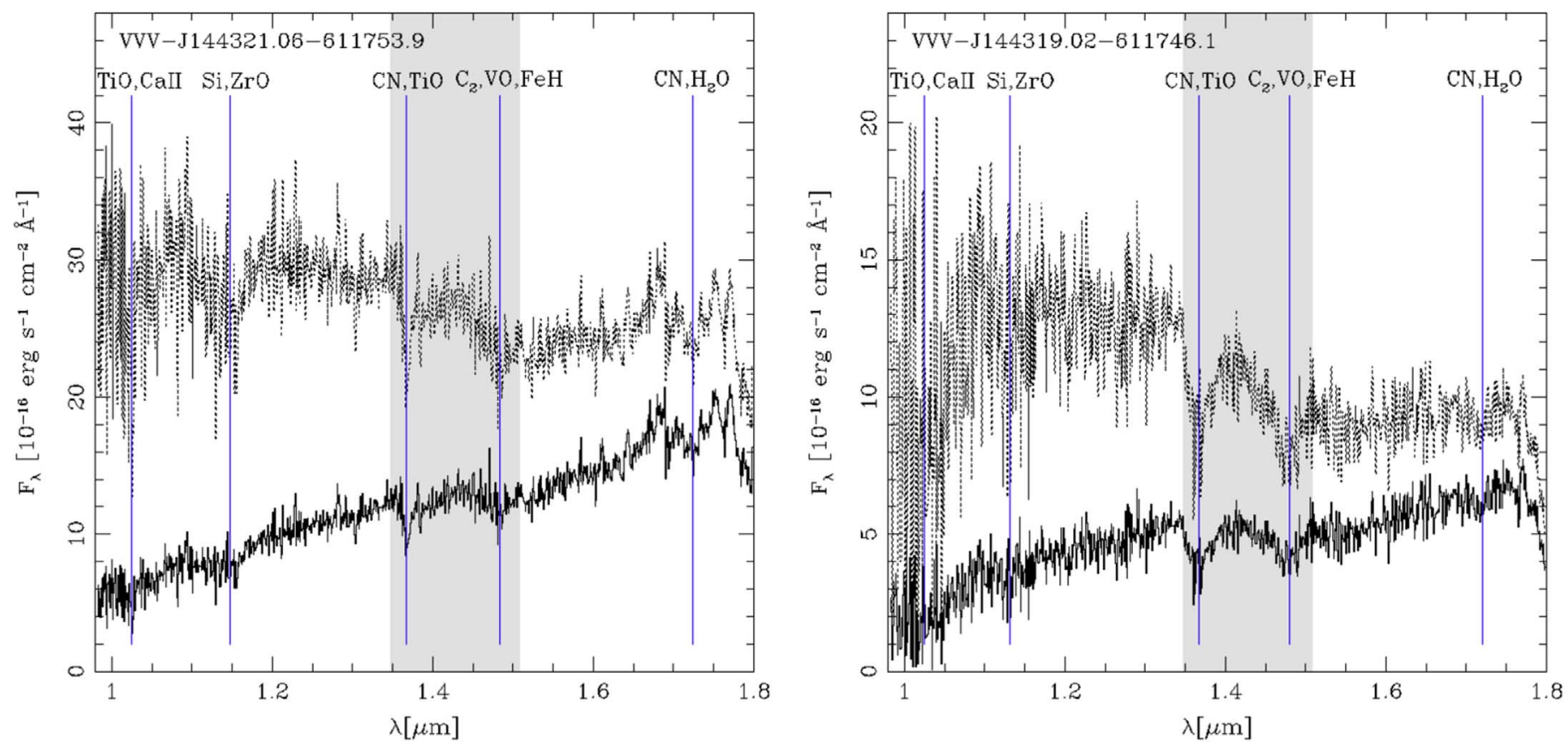

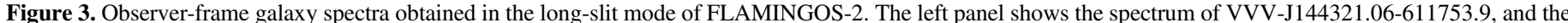

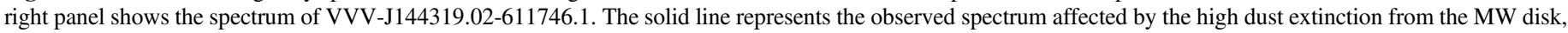

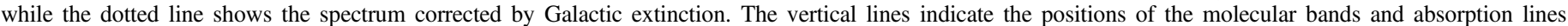
identified in both spectra. The telluric absorption region has been shaded.

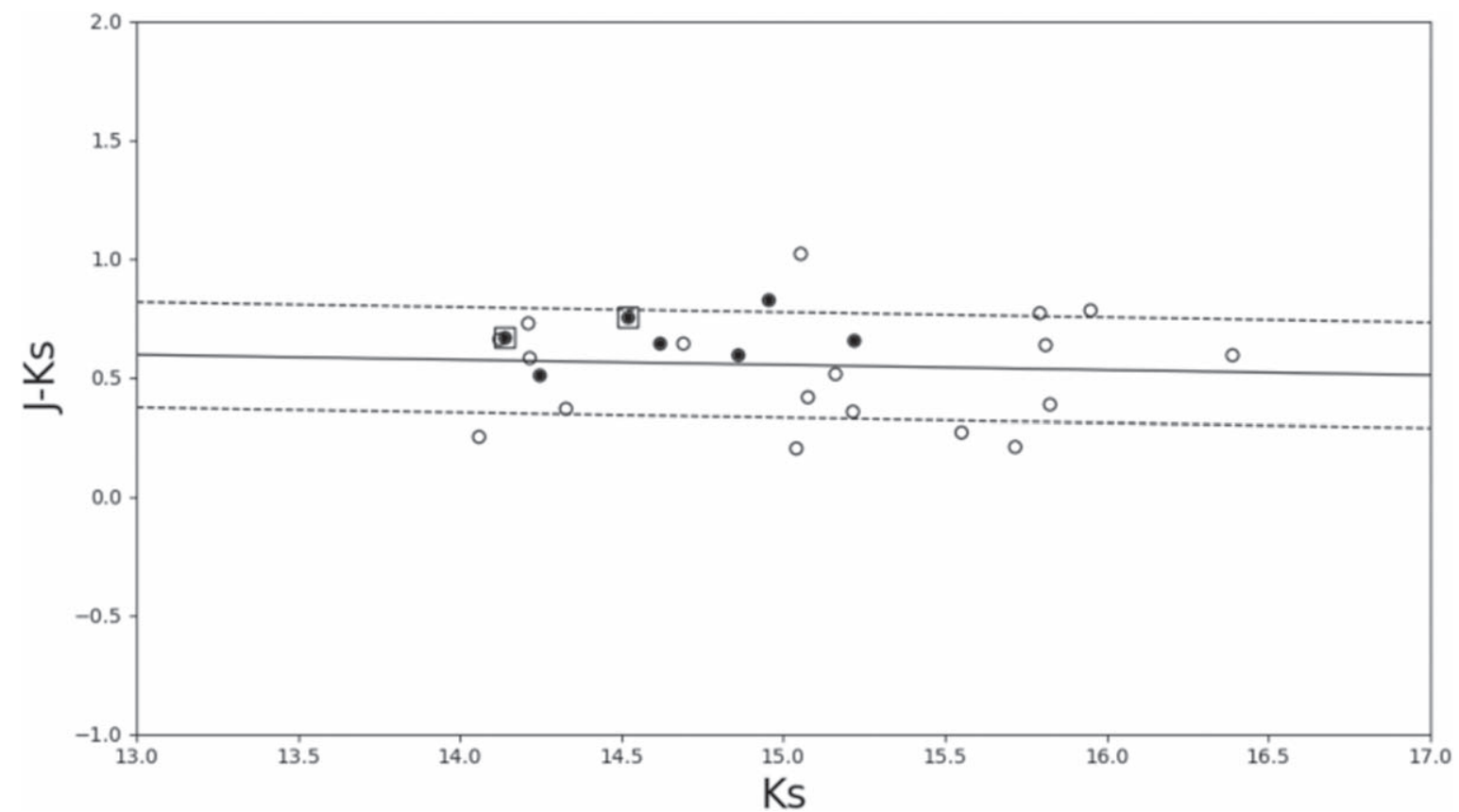

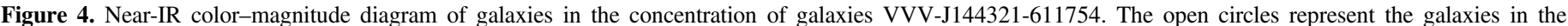

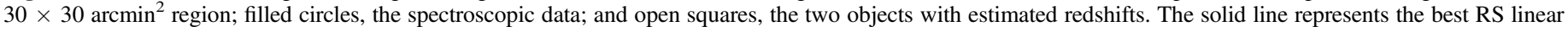
fit; the dashed lines, the $1 \sigma$ dispersion.

\subsection{The Cluster Red Sequence}

In galaxy clusters, the early-type galaxies follow a tight relation in the color-magnitude diagrams called the red sequence (Yee et al. 1999, hereafter RS). We applied the $K$-corrections to the $K_{s}$-band fluxes for the changes of the effective rest-frame wavelengths. We adopted the approximation to the computed observed colors of the 2MASS filters in the Vega system. Following the algorithm by Chilingarian et al. (2010), we used our estimated spectroscopic redshift of the galaxy concentration and the observed $\left(J-K_{s}\right)$ color.

Figure 4 shows the rest-frame near-IR $\left(J-K_{s}\right)$ color $-K_{s}$ magnitude diagram of the 25 galaxies in the $30 \times 30 \operatorname{arcmin}^{2}$ region. The objects with photometric data are represented by open circles, spectroscopic data are represented by filled circles, and the two objects with estimated redshifts are represented by open squares. The cluster RS shows substantial scatter that might be related to the higher $K_{s}$ extinction values 
observed in the central parts of the cluster as compared to outer regions (Section 2.1). The best RS fit relation $\left(J-K_{s}\right)=$ $-0.02 \pm 0.01 \times K_{s}+0.87 \pm 0.05$ was obtained with the 22 galaxies that are also found to belong to group 1 of the clustering analysis. The solid line in the figure represents the best linear fit, and the dashed lines represent the $1 \sigma$ dispersion. Compared with Gladders et al. (1998), who found stable results using $3 \sigma$ iteration, even this conservative RS has about $64 \%$ of the galaxies in the central parts of the galaxy concentration. We obtained similar results using bootstrapping analysis, which supports the idea that these galaxies are part of a galaxy cluster. The cluster RS slope is in agreement with observed near-IR RS for the sample of Stott et al. (2009) and the slope evolution model by Bower et al. (2006) at $z \sim 0.2$.

Finally, we estimated the virial radius of the galaxy cluster at the derived redshift using the method of Merchán \& Zandivarez (2005). We found $R_{\mathrm{vir}}=2.1 \pm 0.4 \mathrm{Mpc}$ for the galaxies in group 1 of the clustering analysis and $R_{\mathrm{vir}}=1.7 \pm 0.2 \mathrm{Mpc}$ for the galaxies that follow the RS within $1 \sigma$. The estimates agree with the typical virial radii of galaxy clusters, and they are comparable within the uncertainties.

\section{Summary}

In this work, we report the first confirmed galaxy cluster, named VVV-J144321-611754, originally detected in the tile d015 of the VVV survey. We performed the photometric procedure in the tile as described in Baravalle et al. (2018), obtaining morphological and photometric parameters of 933 extragalactic sources. We defined a $30 \times 30 \operatorname{arcmin}^{2}$ region centered in the brightest galaxy of the concentration, finding 25 sources that were visually inspected and confirmed to be galaxies. For these galaxies, we obtain extinction-corrected median near-IR colors of $\left(H-K_{s}\right)=0.34 \pm 0.05 \mathrm{mag},(J-H)=$ $0.57 \pm 0.08 \mathrm{mag}$, and $\left(J-K_{s}\right)=0.87 \pm 0.06 \mathrm{mag}$. The median half-light radius is $R_{1 / 2}=1.59 \pm 0 . " 16, \quad C=3.01 \pm 0.08$, $\epsilon=0.30 \pm 0.03$, and Sérsic index $n=4.63 \pm 0.39$. All these morphological parameters are consistent with those of early-type galaxies.

An automatic clustering analysis found four groups in the tile, where the concentration of galaxies VVV-J144321-611754 is a real and the most compact concentration detected, composed of early-type galaxies. Assuming a typical galaxy cluster with low X-ray luminosity, we estimated the photometric redshift of the brightest galaxy to be $z=0.196 \pm 0.025$. The IR spectra of the two brightest galaxies exhibit the typical features of early-type galaxies. The estimated redshift for VVV-J144321.06-611753.9 (the brightest galaxy) is $z=$ $0.234 \pm 0.022$, and that for VVV-J144319.02-611746.1 is $z=0.232 \pm 0.019$. The estimated cluster redshift is $z=$ $0.234 \pm 0.022$. The presence of the cluster RS is clear in the rest-frame color-magnitude diagram of the studied $30 \times 30$ $\operatorname{arcmin}^{2}$ region. Based on all these results, we conclude that the galaxy concentration VVV-J144321-611754 found in the VVV tile d015 is a bona fide galaxy cluster.

This work was partially supported by grants from the Secretaría de Ciencia y Técnica (Secyt) of Universidad Nacional de Córdoba (UNC) and Consejo de Investigaciones Científicas y Técnicas (CONICET). L.D.B. acknowledges the financial support for her PhD thesis from Secyt (UNC) and CONICET. J.L.N.C. is grateful for financial support received from the Programa de Incentivo a la Investigación Académica de la Dirección de Investigación de la Universidad de La Serena (PIA-DIULS), Programa DIULS de Iniciación Científica No. PI15142. J.L.N.C. also acknowledges the financial support from the grant program No. FA9550-15-1-0167 of the Southern Office of Aerospace Research and Development (SOARD), a branch of the Air Force Office of the Scientific Research's International Office of the United States (AFOSR/ IO). F.M.C. acknowledges the financial support from Programa DIDULS PT17145.

We gratefully acknowledge data from the ESO Public Survey program ID 179.B-2002 taken with the VISTA telescope and products from the Cambridge Astronomical Survey Unit (CASU). D.M. is supported by the BASAL Center for Astrophysics and Associated Technologies (CATA) through grant AFB-170002, by the Ministry for the Economy, Development and Tourism, Programa Iniciativa Científica Milenio grant IC120009, awarded to the Millennium Institute of Astrophysics (MAS), and by FONDECYT No. 1170121.

\section{ORCID iDs}

M. V. Alonso (i) https://orcid.org/0000-0001-9684-589X

D. Minniti (i) https://orcid.org/0000-0002-7064-099X

\section{References}

Amôres, E. B., Sodré, L., Minniti, D., et al. 2012, AJ, 144, 127

Baravalle, L. D., Alonso, M. V., Nilo Castellón, J. L., Beamín, J. C., \& Minniti, D. 2018, AJ, 155, 46

Barbá, R. H., Roman-Lopes, A., Nilo Castellón, J. L., et al. 2015, A\&A, 581, A 120

Beamín, J. C., Minniti, D., Gromadzki, M., et al. 2013, A\&A, 557, L8

Borissova, J., Bonatto, C., Kurtev, R., et al. 2011, A\&A, 532, A131

Borissova, J., Ramírez Alegría, S., Chené, A. N., \& Kurtev, R. 2014, RMxAC, 44, 149

Bower, R. G., Benson, A. J., Malbon, R., et al. 2006, MNRAS, 370, 645

Brough, S., Collins, C. A., Burke, D. J., Mann, R. G., \& Lynam, P. D. 2002, MNRAS, 329, L53

Bruzual, G., \& Charlot, S. 2003, MNRAS, 344, 1000

Catelan, M., Minniti, D., Lucas, P. W., et al. 2011, in Carnegie Observatories Astrophysics Ser. 5, RR Lyrae Stars, Metal-Poor Stars, and the Galaxy, ed. A. McWilliam (Pasadena, CA: The Observatories of the Carnegie Institution of Washington), 145

Chilingarian, I. V., Melchior, A.-L., \& Zolotukhin, I. Y. 2010, MNRAS, 405, 1409

Coldwell, G., Alonso, S., Duplancic, F., et al. 2014, A\&A, 569, A49

Conroy, C., Gunn, J. E., \& White, M. 2009, ApJ, 699, 486

Ebeling, H., Mullis, C. R., \& Tully, R. B. 2002, ApJ, 580, 774

Ebeling, H., Voges, W., Bohringer, H., et al. 1996, MNRAS, 281, 799

Gladders, M. D., López-Cruz, O., Yee, H. K. C., \& Kodama, T. 1998, ApJ, 501,571

Henning, P. A., Kraan-Korteweg, R. C., Rivers, A. J., et al. 1998, AJ, 115, 584

Huchra, J. P., Macri, L. M., Masters, K. L., et al. 2012, ApJS, 199, 26

Komatsu, E., Smith, K. M., Dunkley, J., et al. 2011, ApJS, 192, 18

Kraan-Korteweg, R. C., \& Lahav, O. 2000, A\&A Rv, 10, 211

Ma, Y.-Z., Hinshaw, G., \& Scott, D. 2013, ApJ, 771, 137

Maiolino, R., Rieke, G. H., \& Rieke, M. J. 1996, AJ, 111, 537

Mancone, C. L., \& Gonzalez, A. H. 2012, PASP, 124, 606

Maraston, C. 2005, MNRAS, 362, 799

Mason, R., Rodriguez-Ardila, A., Martins, L., et al. 2015, ApJS, 217, 13

McIntyre, T. P., Henning, P. A., Minchin, R. F., Momjian, E., \& Butcher, Z. 2015, AJ, 150, 28

Merchán, M. E., \& Zandivarez, A. 2005, ApJ, 630, 759

Minniti, D., Geisler, D., Alonso-García, J., et al. 2017, ApJL, 849, L24

Minniti, D., Hempel, M., Toledo, I., et al. 2011, A\&A, 527, A81

Minniti, D., Lucas, P. W., Emerson, J. P., et al. 2010, NewA, 15, 433

Mori, H., Maeda, Y., Furuzawa, A., Haba, Y., \& Ueda, Y. 2013, PASJ, 65, 102 
Nevalainen, J., Lumb, D., dos Santos, S., et al. 2001, A\&A, 374, 66

Percival, S. M., Salaris, M., Cassisi, S., \& Pietrinferni, A. 2009, ApJ, 690, 427

Piffaretti, R., Arnaud, M., Pratt, G. W., Pointecouteau, E., \& Melin, J.-B. 2011, A\&A, 534, A109

Ramatsoku, M., Verheijen, M. A. W., Kraan-Korteweg, R. C., et al. 2016, MNRAS, 460, 923

Rayner, J., Cushing, M., Vacca, W., et al. 2009, ApJS, 185, 289

Schlafly, E. F., \& Finkbeiner, D. P. 2011, ApJ, 737, 103
Scrucca, L., Fop, M., Brendan Murphy, T., Adrian, E., \& Raftery, A. E. 2016, The R Journal, 8, 289

Stott, J. P., Pimbblet, K. A., Edge, A. C., Smith, G. P., \& Wardlow, J. L. 2009, MNRAS, 394, 2098

Tully, R. B. 2015, AJ, 149, 54

Voges, W., Aschenbach, B., Boller, T., et al. 1999, A\&A, 349, 389

Yee, H. K. C., Gladders, M. D., \& López-Cruz, O. 1999, in ASP Conf. Ser. 191, Photometric Redshifts and the Detection of High Redshift Galaxies, ed. R. Weymann et al. (San Fransisco, CA: ASP), 166 